\title{
Maspin increases Ku70 acetylation and Bax-mediated cell death in cancer cells
}

\author{
SOOK-JA LEE, HAERIM JANG and CHAEHWA PARK \\ Cancer Genetics Laboratory, Biomedical Research Institute, Samsung Medical Center, \\ Sungkyunkwan University School of Medicine, Seoul 135-710, Republic of Korea
}

Received September 2, 2011; Accepted October 7, 2011

DOI: $10.3892 /$ ijmm.2011.833

\begin{abstract}
Ku70, a DNA repair protein, was recently identified as a critical anti-apoptotic protein that inhibits Bax translocation to mitochondria. The dissociation of Bax from $\mathrm{Ku} 70$ is essential for the apoptotic activity of Bax. Here, we show that maspin, a tumor suppressor protein frequently lost in cancer, regulates this process. Maspin increased cell death in a Ku70 acetylation-dependent manner. Maspin inhibited histone deacetylase 1 (HDAC1) and thus increased the acetylation of $\mathrm{Ku} 70$ and the dissociation of Bax from $\mathrm{Ku} 70$, which led to the induction of apoptosis. These results reveal maspin as a $\mathrm{Ku} 70$-interacting molecule and provide the basis for a new endogenous acetylation-based control mechanism that reduces $\mathrm{Ku} 70$-mediated sequestration of Bax from mitochondria.
\end{abstract}

\section{Introduction}

Cancer cells are often defective in the ability to induce cell death in response to intra- or extracellular stress. In several in vivo studies, tumors that expressed maspin showed suppressed growth and progression (1-4). The first in vitro evidence that maspin can promote cell death reported that maspin sensitized breast carcinoma cells to staurosporine (STS)-induced apoptosis (5). More recently, others have reported that maspin inhibited tumor progression by enhancing cellular sensitivity to apoptotic stimuli via modulation of a Bax-mediated mitochondrial permeability transition (6-8).

Ku70 was initially reported as a DNA repair protein (9). Subsequently, Sawada et al (10) identified the Bax-binding property of Ku70 by screening a human cDNA library and demonstrated that $\mathrm{Ku} 70$ interacts with Bax through the Ku70 $\mathrm{C}$-terminal domain. $\mathrm{Ku} 70$ is an acetylation-sensitive inhibitor of Bax, and the lysine residues subject to acetylation lie in a

Correspondence to: Dr Chaehwa Park, Biomedical Research Institute, Samsung Medical Center, Sungkyunkwan University School of Medicine, 50 Irwon-dong, Seoul 135-710, Republic of Korea

E-mail: cpark@skku.edu

Key words: Ku70, maspin, acetylation, Bax, apoptosis
C-terminal linker domain adjacent to the region responsible for Bax interaction. Histone deacetylase (HDAC) inhibitors were shown to increase acetylation of $\mathrm{Ku} 70$, suggesting that the Bax-inhibitory function of $\mathrm{Ku} 70$ may be modulated by HDACs (11). Specifically, mutations of Ku70, K539Q and K542Q, which mimic acetylated lysine, rendered Ku70 unable to inhibit Bax. As Ku70 is a constitutively expressed protein, specific cytoplasmic proteins may control the $\mathrm{Ku} 70$-Bax interaction and thus, indirectly, the Bax-mediated mitochondrial apoptosis. Previously, maspin was reported to interact with histone deacetylase 1 (HDAC1) and inhibit its activity (12).

In the present study, we show for the first time that maspin increases acetylation of $\mathrm{Ku} 70$ and thus releases Bax from Ku70-mediated inhibition. Significantly, our results suggest that cell death suppression by Ku70 can be blocked by maspin by inhibition of HDAC1, which represents another important regulatory pathway upstream of Bax. This interaction of maspin with $\mathrm{Ku} 70$ and HDAC1 may provide a fine control mechanism for mitochondrial cell death and chemosensitivity in epithelial cancer.

\section{Materials and methods}

Cell culture and transfection. NCI-H157 cells were grown in RPMI (Gibco Life Science, Grand Island, NY). HeLa and 293T cells were grown in DMEM (Gibco). Cells were transfected using Effectene (Qiagen, Valencia, CA). To examine the effects of Ku70 and maspin on cell death, $2 \times 10^{6}$ cells were transfected with pEGFP (GFP or GFP-Ku70 expression plasmid, Clontech, Palo Alto, CA) and pCMV-Tag4C (mycmaspin expression plasmid, Clontech).

Yeast two-hybrid analysis. The maspin bait sequence (amino acids 38-375) was inserted into the pLexA DNA-binding domain vector (pBD; Clontech) to make pBD-maspin. The reporter strain EGY48 (Clontech) was sequentially transformed with pBD-maspin and a pB42 AD-tagged HeLa cDNA library. Screening was performed according to the protocol provided with the Matchmaker two-hybrid system (Clontech), and positive clones were selected on supplemented minimal galactose medium. As an additional check of the positive colonies, qualitative blue-white screening with $40 \mu \mathrm{g} / \mathrm{ml}$ 5-bromo-4-chloro-3-indolyl-D-galactopyranoside (X-gal) was performed. Control strains, comprised of pJG4-5/Daxx 
Table I. Primers used for PCR.

\begin{tabular}{lll}
\hline Gene & & \multicolumn{1}{c}{ Primer sequence } \\
\hline Ku70 (aa 1-609) & Forward & 5'-AATGGATCCGCCACCATGTCAGGGTGGGAGTCATAT-3' \\
& Reverse & 5'-CGGCCTCGAGTCAGTCCTGGAAGTGCTT-3' \\
Ku70 (aa 1-370) & Forward & 5'-AATGGATCCGCCACCATGTCAGGGTGGGAGTCATAT-3' \\
& Reverse & 5'-GAACTCGAGTTACTTTATTGGAGGAGGCT-3' \\
Ku70 (aa 370-609) & Forward & 5'-AATGGATCCGCCACCATGCCAGAGGAGTCGCTGGTG-3' \\
& Reverse & 5'-CGGCCTCGAGTCAGTCCTGGAAGTGCTT-3' \\
Ku70 (aa 500-609) & Forward & 5'-AATGGATCCGCCACCATGCCGGAACAAGCAGTGGAC -3' \\
& Reverse & 5'-CGGCCTCGAGTCAGTCCTGGAAGTGCTT-3' \\
Maspin R340A & Forward & 5'-AGGTGCCAGGAGCAGCGATCCTGCAGCAC-3' \\
& Reverse & 5'-GTGCTGCAGGATCGCTGCTCCTGGCAC-3' \\
& &
\end{tabular}

and pLexA/sentrin (positive control; P) and pJG4-5/FADD and LexA/sentrin (negative control; N), both in EGY48, were streaked on SD medium lacking uracil and histidine (25).

Plasmids. All constructs were generated by PCR using primers designed from the coding region of the relevant human cDNAs (Table I). Full-length Ku70 (GeneBank accession No. U04313) was generated from HeLa mDNA by reverse transcription-PCR (RT-PCR) and cloned into pcDNA3.1 (Invitrogen, Carlsbad, $\mathrm{CA}$, pCMV-Tag4C or pEGFP. For in vitro transcription and translation, the full-length $\mathrm{Ku} 70$ and its deletion constructs were subcloned into pcDNA3.1(+). Myc-tagged-maspin (18) was cloned into pCMV-Tag4C. For the detection of binding between maspin and in vitro translated Ku70, maspin was cloned, in frame, into pGEX4T-1 (Amersham Pharmacia Biotech, UK). Maspin mutants, P176S and R340A were cloned into pCMV-Tag4C and pEGFP, respectively.

In vitro transcription, translation, and GST pull-down assay. Binding between maspin and Ku70 in vitro was assessed using glutathione S-transferase (GST) fusion proteins. GST fused maspin protein was expressed in E. coli BL21 (DE3) with IPTG (isopropyl-B-D-thiogalactopyranoside) induction and obtained by cell sonication. Full-length Ku70 and deletion proteins were translated in vitro with the TNT transcription/translation system kit (Promega, Madison, WI). The bound proteins were captured by glutathione-Sepharose beads and resolved using SDS-PAGE.

Immunoprecipitation and immunoblotting. To test for an association between endogenous maspin and Ku70 proteins, HeLa cells $(10 \mathrm{~cm}$ dish) were lysed in $200 \mu 1$ of lysis buffer (50 mM Tris- $\mathrm{HCl}, \mathrm{pH} 8.0,150 \mathrm{mM} \mathrm{NaCl}, 1 \mathrm{mM}$ EDTA, $1 \%$ NP-40, 0.4 mM PMSF) including a protease inhibitor cocktail (Promega). Immunoprecipitation was conducted using indicated antibodies. Beads were washed and bound proteins were detected by immunoblotting as reported previously (18).

Immunofluorescence. HeLa cells $\left(2 \times 10^{5}\right)$ were transfected with pEGFP-Ku70 and pRFP-maspin, using $1 \mu \mathrm{g}$ of each plasmid. The cells were then stained with DAPI, and examined with an Olympus laser scanning confocal microscope.

\section{Results}

Maspin interacts with Ku70. To understand the mechanism underlying the Bax-activating function of maspin, we initially searched for maspin-binding proteins in a human HeLa cDNA library, using a yeast two-hybrid screening method. DNA-encoding maspin (aa 38-375) was cloned into the pLexA vector to construct a bait plasmid. A yeast two-hybrid assay identified several proteins that interact with maspin, including Ku70. DNA sequencing and basic alignment searches of the NCBI database showed that one of the positive clones encoded aa 278-609 of Ku70 (Fig. 1A). To confirm whether maspin and Ku70 interact in mammalian cells, a complementary immunoprecipitation experiment was performed with endogenous proteins extracted from HeLa cells. As shown in Fig. 1B, Ku70 was detected by Western blotting analysis after co-precipitation with maspin.

Assays performed in vitro showed that maspin pulled down Ku70 and vice versa (Fig. 1C and D), suggesting an interaction between maspin and Ku70. GST-maspin interacted with $\mathrm{Ku} 70$, but not with an $\mathrm{N}$-terminal Ku70 construct encoding aa 1-300 $\left(\mathrm{Ku}^{11-300}\right)$, which contains a DNA-binding domain. Although the C-terminal Ku70 (Ku70 $\left.{ }^{370-609}\right)$ interacted with in vitro-translated maspin, a smaller Ku70 construct encoding the Bax-binding domain $\left(\mathrm{Ku} 70^{500-609}\right)$ could not pull down maspin (Fig. 1D). These results indicate that maspin interacts with Ku70 via a C-terminal region of Ku70, known to include critical acetylation sites and a Bax-binding domain (11).

Maspin colocalizes with Ku70 in the cytoplasm. To examine the association of maspin with Ku70 in vivo, the localization of maspin and $\mathrm{Ku} 70$ was observed by confocal microscopic imaging of proteins in HeLa cells. Whereas endogenous Ku70 is expressed at a high level, maspin expression levels are usually down-regulated in cancer cells. Thus, to compare the immunofluorescent intensities of maspin and Ku70, HeLa cells were co-transfected with recombinant maspin and Ku70, fused with RFP and GFP, respectively. Ku70 proteins were distributed in the nucleus and cytoplasm, whereas maspin proteins were localized mainly in the cytoplasm (Fig. 2A). The intracellular locations of maspin and Ku70 were further examined 
A

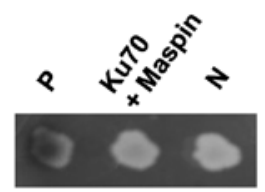

C Input $\quad \frac{\text { Pull Down }}{\text { GST GST-Maspin }}$

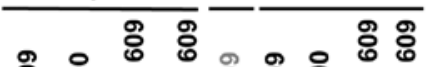

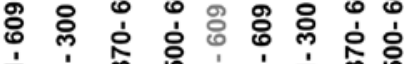
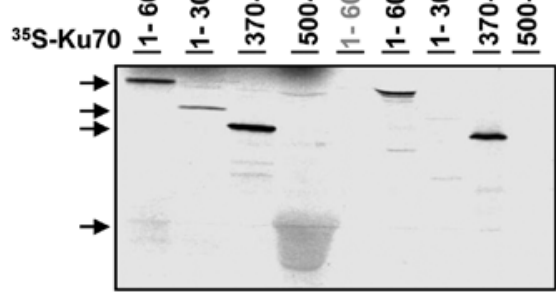

B

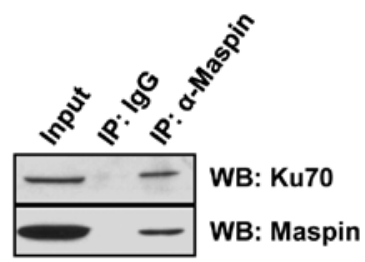

Figure 1. Maspin interacts with Ku70, in vitro and in vivo. (A) A yeast two-hybrid system was used to analyze the interaction between maspin and Ku70. To evaluate the interactions between maspin and Ku70, both maspin (aa 38-375) and Ku70 (aa 278-609) were expressed, as pLexA and pJG4-5 fusion proteins, respectively, in yeast. Transformants were streaked onto SD medium containing X-gal. P, positive control. N, negative control. (B) Co-immunoprecipitation of endogenous maspin with Ku70. Total cell lysates from HeLa cells were immunoprecipitated with anti-maspin antibody and the presence of Ku70 in the immunoprecipitates was detected by Western blotting using an anti-Ku70 antibody. Total cell lysate was loaded as input. (C) In vitro binding of GST fused recombinant maspin to full-length or truncated ${ }^{35} \mathrm{~S}$-Met Ku70. In vitro translated Ku70 was pulled down with GST or GST-fused recombinant maspin. Autoradiography after SDS-PAGE shows the in vitro interaction between maspin and Ku70. (D) In vitro binding of GST-fused recombinant Ku70 to in vitro translated ${ }^{35} \mathrm{~S}-$ Met maspin. CBB, Coomassie brilliant blue stained GST fusion proteins from the same gel were aligned to show protein levels.

A

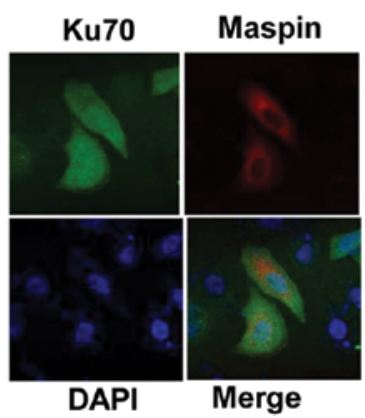

B

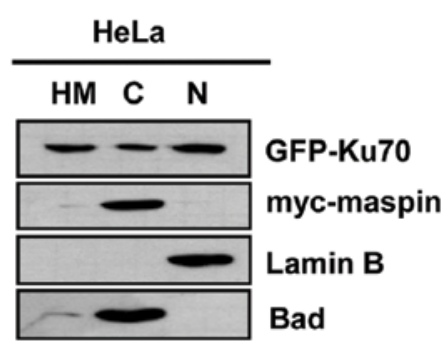

C

293T

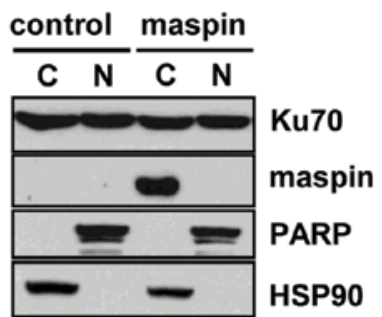

Figure 2. Maspin colocalizes with Ku70 in the cytoplasm. (A) Confocal microscope image indicating cytoplasmic colocalization of maspin with Ku70 in HeLa cells. Cells were transfected with pEGFP-Ku70 and pRFP-maspin. Merged image of GFP-tagged Ku70 (green), RFP-tagged maspin (red) and DAPI-stained DNA (blue) clearly shows colocalization of maspin with Ku70. (B) Subcellular fractionation and Western blotting analysis of HeLa cell lysates. The purity of each fraction was ascertained by re-probing the blot for nuclear and cytoplasmic markers (Lamin B and Bad, respectively). HM, heavy membrane; C, cytosol; N, nucleus. (C) Subcellular fractionation and Western blotting analysis of maspin-negative 293T cells. Cells transfected with control vector or maspin were lysed and subcellular fractionation was performed. PARP and HSP90 were used as nuclear and cytoplasmic markers, respectively.

by subcellular fractionation and Western blotting analysis in HeLa (Fig. 2B) and maspin-transfected 293T cells (Fig. 2C). Consistent with the immunoprecipitation data and confocal microscopic images, coexpression of $\mathrm{Ku} 70$ and maspin was detected in the cytosolic fraction. Together, these results indicate that interaction of maspin with $\mathrm{Ku} 70$ may take place in the cytoplasm but not in the nucleus.

Maspin increases lysine-acetylation of Ku70 and inhibits endogenous Ku70-Bax interaction. To assess whether maspin interacts with $\mathrm{Ku} 70$ and mediates $\mathrm{Ku} 70$ acetylation in lung cancer cells, we transfected maspin-deficient NCI-H157 cells with either the maspin expression plasmid, or empty vector. Immunoprecipitation of maspin, followed by probing of the immunocomplex with an antibody against $\mathrm{Ku} 70$, revealed that maspin interacts with Ku70 (Fig. 3A). In addition, reverse immunoprecipitation with antibody against $\mathrm{Ku} 70$, followed by probing of the immunocomplex with anti-maspin antibody, further confirmed the Ku70-maspin interaction (Fig. 3B).

Previously, HDAC inhibitors have been shown to increase acetylation of $\mathrm{Ku} 70$ (11). In addition, HDAC1, a major HDAC responsible for histone deacetylation, has been identified as a maspin-interacting molecule in a yeast two-hybrid screening project (12). In the same report, an inhibitory effect of maspin on HDAC1 activity was proposed, involving direct molecular interaction. Thus, we investigated whether maspin may interact with $\mathrm{HDAC} 1$ and increase $\mathrm{Ku} 70$ acetylation. As shown in Fig. 3A, immunoprecipitation of maspin, followed 
A

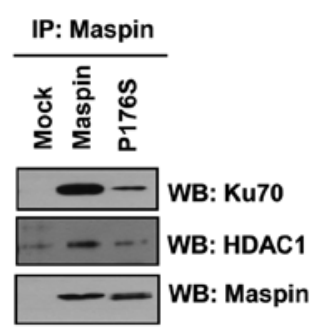

B

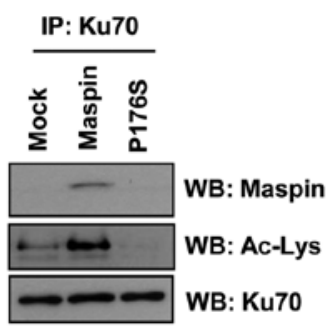

C

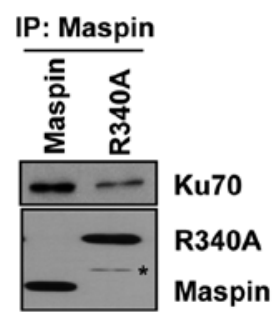

D

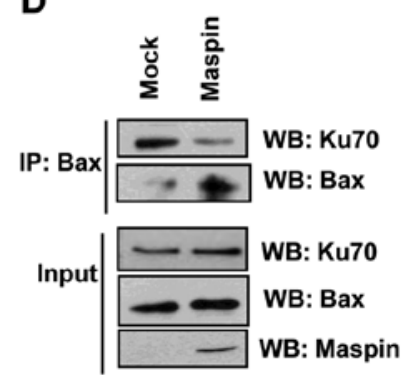

Figure 3. In vivo interaction of maspin with endogenous target proteins in NCI-H157 lung cancer cells transfected with empty vector (mock), maspin or mutant maspin (P176S). (A) Total cell lysates were immunoprecipitated with an anti-maspin antibody and the presence of Ku70 and HDAC1 in the immunoprecipitates was detected with anti-Ku70 antibody and anti-HDAC1 antibody, respectively. (B) Total cell lysates were immunoprecipitated with an anti-Ku70 antibody and the presence of maspin and acetylated lysine in the immunoprecipitates was detected with anti-maspin antibody and anti-acetyl lysine antibody, respectively. (C) In vivo interaction of recombinant maspin with endogenous Ku70 in NCI-H157 cells transfected with wild type maspin or mutant maspin (R340A). Total cell lysates were immunoprecipitated with an anti-maspin antibody and the presence of Ku70 in the immunoprecipitates was detected with anti-Ku70 antibody. (D) Total cell lysates were immunoprecipitated with an anti-Bax antibody and the presence of Ku70 in the immunoprecipitates was detected with an anti-Ku70 antibody. The total cell lysate was loaded as input and analyzed by immunoblotting with the indicated antibodies on the right.

A
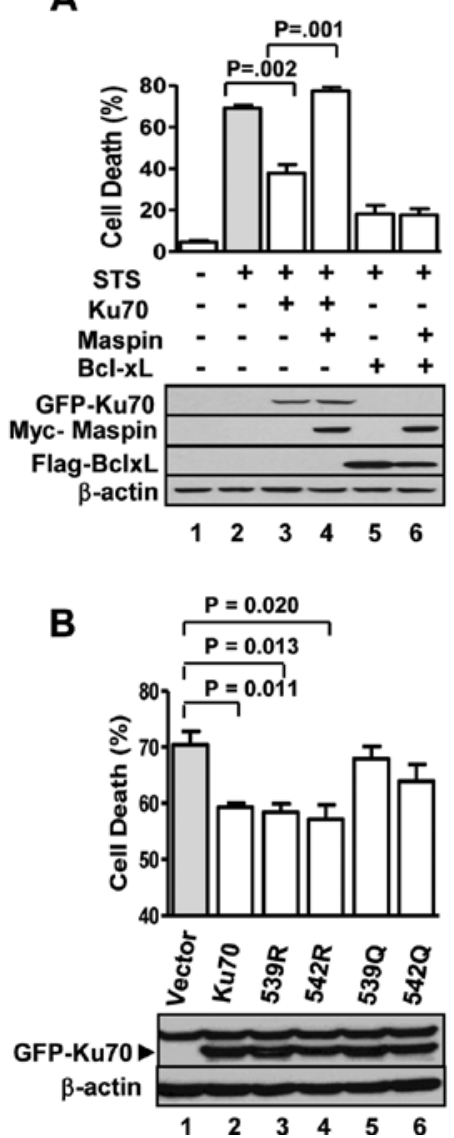

$\mathrm{NCl-H157}$

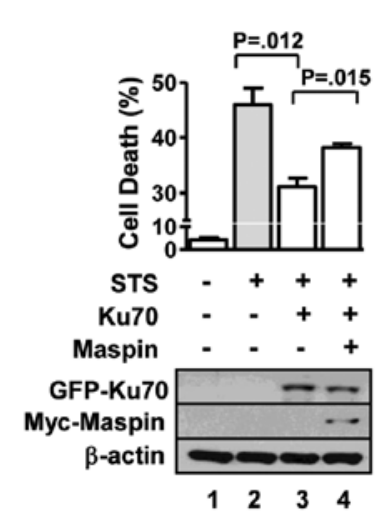

C

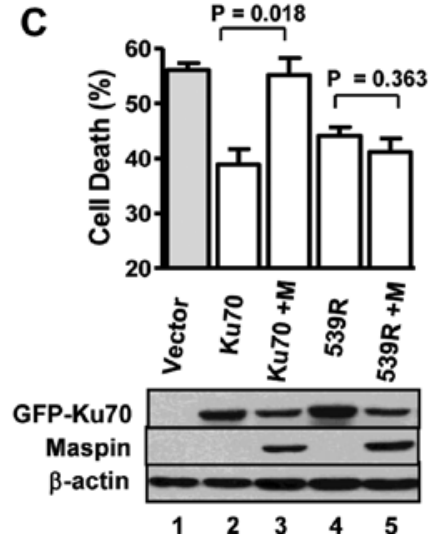

Figure 4. Acetylation residue of Ku70 is critical for maspin-induced restoration of cell death. (A) HeLa, NCI-H157, and 293T cells were transfected with the indicated expression vectors. One day post-transfection, the cells were treated with staurosporine ( $200 \mathrm{nM}$ for HeLa or $400 \mathrm{nM}$ for NCI-H157 cells) or etoposide $(100 \mu \mathrm{M}$ for $293 \mathrm{~T})$ for $24 \mathrm{~h}$. After the treatment, cells were stained with Hoechst (HeLa) to score the percentage of cells undergoing apoptosis. NCI-H157 and 293T cells were stained with trypan blue solution and the blue-stained dead cells were counted under a microscope. (B) The Ku70-mediated inhibition of cell death was varied depending on the mutation of lysines at aa 539 and 542. (C) Maspin could not enhance the etoposide-induced death of 293T cells expressing Ku70 acetylation-resistant mutants. The values shown represent the mean of three independent experiments (P values, t-test; bars, SEM). Cells were evaluated for the level of recombinant protein expression by immunoblotting analysis using GFP (Ku70), myc (maspin), and FLAG (Bcl-xL) antibodies.

by probing of the immunocomplex with anti-HDAC1 antibody, revealed that maspin interacts with HDAC1. Moreover, the immunoprecipitation of $\mathrm{Ku} 70$, followed by probing of the immunocomplex with an antibody against acetylated lysine, confirmed that lysine-acetylation of $\mathrm{Ku} 70$ is increased in maspin-expressing cells (Fig. 3B).

Previously, we have reported that a maspin mutation (P176S), frequently found in cancer, decreased apoptosis and 
increased colony formation (13). As shown in Fig. 3, maspin (P176S) interacts with Ku70 much less effectively than wildtype maspin and does not increase Ku70 acetylation. Another maspin mutant protein (R340A), with an aa substitution of Arg340 to Ala at the putative $\mathrm{P}_{1}$ site of the reactive site loop, lost tumor suppressor function (14). R340A weakly binds to Ku70 compared to the wild-type protein (Fig. 3C), suggesting that the reactive site loop sequence of maspin may be important for this interaction. As acetylation of Ku70 controls Bax-mediated apoptosis (11), we investigated whether $\mathrm{Ku} 70$ binding to Bax may be affected by maspin. Ku70 was pulled down by antiBax antibody, but the level of Ku70 in the immunoprecipitate was also dramatically decreased in maspin-expressing cells (Fig. 3D).

Maspin restores Ku70-suppressed cell death. To examine the functional significance of the maspin-Ku70 interaction, maspin and Ku70 was co-transfected into HeLa cells. Ectopic overexpression of maspin restored $\mathrm{Ku} 70$-mediated cell death inhibition (Fig. 4A, left; lane 3 vs. 4). To test the specificity of maspin for Ku70, we used a Bcl-xL overexpression system to explore whether maspin can modulate the activity of Bcl-xL. However, maspin did not affect the Bcl-xL-mediated inhibition of cell death (Fig. 4A, left; lane 5 vs. 6). For validation, we performed the same experiment using maspin-deficient NCI-H157 (Fig. 4A, middle) and 293T cells (Fig. 4A, right). Overexpression of $\mathrm{Ku} 70$ decreased cell death in both cell lines (lane 2 vs. $3, \mathrm{P}<0.02$ ). Moreover, maspin overexpression restored the Ku70-mediated decrease of cell death in this system (lane 3 vs. $4, \mathrm{P}<0.02$ ), whereas mutant maspin P176S (13), with an even higher level of expression than wild-type maspin, could not restore $293 \mathrm{~T}$ cell death in a parallel experiment (Fig. 4A, right ; lane 3 vs. 5).

Maspin-induced cell death is associated with Ku70 acetylation. When Bax is activated by cell death-inducing agents, non-acetylated $\mathrm{Ku} 70$ binds and sequesters Bax, thus reducing mitochondrial translocation (11). Based on our observation that maspin interacts with HDAC1 and increases Ku70 acetylation (Fig. 3), we hypothesized that acetylation of $\mathrm{Ku} 70$ may be associated with maspin-mediated modulation of cell death. To test this idea, we constructed a Ku70 mutant in which Lys-539 and Lys-542, critical acetylation-sensitive sites for Ku70-Bax binding, were replaced with arginine residues (K539R and K542R, respectively), to prevent acetylation. We next tested the ability of the Ku70 mutant to inhibit cell death, and explored whether the cell death-inhibitory function was blocked by maspin. Specifically, GFP-Ku70 wild-type or GFP-Ku70 mutant (K539R and K542R) constructs, or vector alone, were transfected into $293 \mathrm{~T}$ cells, with or without cotransfection of maspin. Trypan blue staining indicated that overexpression of wild type, K539R or K542R significantly suppressed cell death (Fig. 4B; lane 1 vs. 2, 3 or 4, $\mathrm{P}<0.02$ ). However, the acetylationmimicking mutants K539Q and K542Q could not suppress cell death, compared with cells receiving vector alone (Fig. 4B). Together, these data indicate that lysine acetylation plays a critical role in $\mathrm{Ku} 70$-mediated suppression of cell death. In addition, to investigate the importance of the acetylation site in maspin-mediated Ku70 inhibition, we performed co-transfections of maspin and the acetylation-resistant mutants, K539R and K542R, into 293T cells. Interestingly, maspin could not modulate cell death of $293 \mathrm{~T}$ cells that carried a mutation in the critical acetylation site of $\mathrm{Ku} 70$, indicating that acetylation of $\mathrm{Ku} 70$ might be critical in maspin-mediated cell death stimulation (Fig. 4C).

\section{Discussion}

Apoptosis is a key tumor suppressive mechanism and can be initiated by activation of the pro-apoptotic factor Bax. Ku70, a DNA repair protein, has recently been shown to suppress apoptosis by sequestering Bax from mitochondria $(10,11)$. However, the mode of activation of the death effector Bax in the presence of the ubiquitous Bax inhibitor $\mathrm{Ku} 70$ has not been clear. Our study provides the first evidence for a novel inhibitory effect on Ku70 by maspin.

Previously, we and others have found that maspin sensitizes cancer cells to cell death $(5-8,13)$. The present study shows that maspin interacts with $\mathrm{Ku} 70$ and the results support the Bax-inhibitory activity of Ku70. Specifically, binding of maspin and Ku70 was analyzed by yeast-two-hybrid analysis and co-immunoprecipitation, and the results support the possibility of a direct interaction between the 2 proteins. In addition, we found that $\mathrm{Bax}$ is dissociated from $\mathrm{Ku} 70$ in the presence of maspin, which may be explained by the direct interaction of Ku70 with maspin and the maspin-mediated increase in $\mathrm{Ku} 70$ acetylation, which, in turn, abolishes the Bax-inhibitory activity of $\mathrm{Ku} 70$.

Maspin was found to increase acetylation of Ku70 (Fig. 3). This phenotype of maspin may be explained by the previously reported HDAC-targeting activity of maspin (12). Li et al (12) proposed that maspin had an inhibitory effect on HDAC1, but not on other class I HDACs (e.g., HDAC2 and HDAC3). Treating cells with deacetylase inhibitors abolishes the ability of $\mathrm{Ku} 70$ to suppress Bax-mediated apoptosis (15). Our data support this hypothesis by demonstrating that maspin increases Lys-acetylation of Ku70 and blocks Ku70 binding to Bax. Maspin-mediated acetylation of Ku70 also supports the previously-reported findings of maspin-induced Bax activation (6). Cohen et al showed that acetylation of the Ku70 C-terminus controlled Bax-mediated apoptosis (11). Our data support this hypothesis by demonstrating that the Ku70 mutants, K539R and K542R, which mimic the non-acetylated state of the wild type protein (16), were not sensitive to maspin. Although we do not have sufficient evidence to conclude whether direct binding of maspin and $\mathrm{Ku} 70$ is critical for inhibition of $\mathrm{Ku} 70$, our data clearly show that maspin increases the release of Bax from $\mathrm{Ku} 70$ and restores $\mathrm{Ku} 70$-mediated cell death inhibition. Future biochemical analyses using purified proteins may help to elucidate the sequence of interactions among Ku70, HDACs, acetyltransferases, Bax, and maspin.

Importantly, as maspin is an epithelium-specific gene (17), maspin may act as an epithelium-specific inhibitor of $\mathrm{Ku} 70$. We have shown that cytoplasmic expression of maspin in lung cancer cells is suppressive of the tumor $(13,18)$. It has also been shown that cancer patients who express higher levels of maspin respond better to chemotherapy (19). Ku70 expression correlates inversely with sensitivity to conventional therapy (20); a decrease in Ku70 is known to enhance sensitivity to chemotherapy and radiation $(21,22)$. Given that maspin inhibits 
Ku70, maspin-induced sensitization of cancer cells to cell death $(5-8,13)$ may not be surprising. However, considering the ubiquitous existence and pivotal role of $\mathrm{Ku} 70$ in vivo $(23,24)$, it is likely that $\mathrm{Ku} 70$ inhibition of maspin may be tightly regulated by subcellular localization and modulatory proteins including HDACs.

In summary, we report for the first time, that maspin interacts with $\mathrm{Ku} 70$, increases $\mathrm{Ku} 70$ acetylation, and decreases interaction of $\mathrm{Ku} 70$ with Bax. Thus, maspin may inactivate $\mathrm{Ku} 70$ by acting as an endogenous HDAC1 inhibitor. As HDAC inhibitors are known to have tumor suppressor activity, the HDAC inhibitory activity of maspin might, in part, account for maspin tumor suppressor function. However, it remains to be determined how interaction of Ku70 and maspin may influence other events including DNA repair and the developmental process. Our data suggest that maspin increases cell death through inhibition of $\mathrm{Ku} 70$, in addition to the previously known tumor-suppressive roles of maspin in angiogenesis and metastasis.

\section{Acknowledgements}

This study was supported by grants from the Korea Research Foundation (KRF-2011-0016973) and the Samsung Biomedical Research Institute (SBRI P-B0-001).

\section{References}

1. Cher ML, Biliran HR Jr, Bhagat S, Meng Y, Che M, Lockett J, Abrams J, Fridman R, Zachareas M and Sheng S: Maspin expression inhibits osteolysis, tumor growth, and angiogenesis in a model of prostate cancer bone metastasis. Proc Natl Acad Sci USA 100: 7847-7852, 2003

2. Zhang M, Shi Y, Magit D, Furth PA and Sager R: Reduced mammary tumor progression in WAP-TAg/WAP-maspin bitransgenic mice. Oncogene 19: 6053-6058, 2000.

3. Sheng S, Carey J, Seftor EA, Dias L, Hendrix MJ and Sager R: Maspin acts at the cell membrane to inhibit invasion and motility of mammary and prostatic cancer cells. Proc Natl Acad Sci USA 93: 11669-11674, 1996.

4. Zou Z, Anisowicz A, Hendrix MJ, Thor A, Neveu M, Sheng S, Rafidi K, Seftor E and Sager R: Maspin, a serpin with tumorsuppressing activity in human mammary epithelial cells. Science 263: 526-529, 1994.

5. Jiang N, Meng Y, Zhang S, Mensah-Osman E and Sheng S: Maspin sensitizes breast carcinoma cells to induced apoptosis. Oncogene 21: 4089-4098, 2002.

6. Latha K, Zhang W, Cella N, Shi HY and Zhang M: Maspin mediates increased tumor cell apoptosis upon induction of the mitochondrial permeability transition. Mol Cell Biol 25: $1737-1748,2005$

7. Zhang W, Shi HY and Zhang M: Maspin overexpression modulates tumor cell apoptosis through the regulation of Bcl-2 family proteins. BMC Cancer 5: 50, 2005.

8. Liu J, Yin S, Reddy N, Spencer C and Sheng S: Bax mediates the apoptosis-sensitizing effect of maspin. Cancer Res 64: 1703-1711, 2004.
9. Bliss TM and Lane DP: Ku selectively transfers between DNA molecules with homologous ends. J Biol Chem 272: 5765-5773, 1997.

10. Sawada M, Sun W, Hayes P, Leskov K, Boothman DA and Matsuyama S: Ku70 suppresses the apoptotic translocation of Bax to mitochondria. Nat Cell Biol 5: 320-329, 2003.

11. Cohen HY, Lavu S, Bitterman KJ, Hekking B, Imahiyerobo TA, Miller C, Frye R, Ploegh H, Kessler BM and Sinclair DA: Acetylation of the $\mathrm{C}$ terminus of $\mathrm{Ku} 70$ by $\mathrm{CBP}$ and PCAF controls Bax-mediated apoptosis. Mol Cell 13: 627-638, 2004.

12. Li X, Yin S, Meng Y, Sakr W and Sheng S: Endogenous inhibition of histone deacetylase 1 by tumor-suppressive maspin. Cancer Res 66: 9323-9329, 2006.

13. Jang HL, Nam E, Lee KH, Yeom S, Son HJ and Park C: Maspin polymorphism associated with susceptibility to apoptosis and in vivo tumorigenesis. Int J Mol Med 22: 333-338, 2008.

14. Ngamkitidechakul C, Warejcka DJ, Burke JM, O'Brien WJ and Twining SS: Sufficiency of the reactive site loop of maspin for induction of cell-matrix adhesion and inhibition of cell invasion. J Biol Chem 278: 31796-31806, 2003.

15. Subramanian C, Opipari AW Jr, Bian X, Castle VP and Kwok RP: $\mathrm{Ku} 70$ acetylation mediates neuroblastoma cell death induced by histone deacetylase inhibitors. Proc Natl Acad Sci USA 102: 4842-4847, 2005

16. Li M, Luo J, Brooks CL and Gu W: Acetylation of p53 inhibits its ubiquitination by Mdm2. J Biol Chem 277: 50607-50611, 2002.

17. Futscher BW, Oshiro MM, Wozniak RJ, Holtan N, Hanigan CL, Duan $\mathrm{H}$ and Domann FE: Role for DNA methylation in the control of cell type specific maspin expression. Nat Genet 31: 175-179, 2002.

18. Kim S, Han J, Kim J and Park C: Maspin expression is transactivated by p63 and is critical for the modulation of lung cancer progression. Cancer Res 64: 6900-6905, 2004.

19. Dietmaier W, Bettstetter M, Wild PJ, Woenckhaus M, Rümmele P, Hartmann A, Dechant S, Blaszyk H, Pauer A, Klinkhammer -Schalke M and Hofstädter F: Nuclear maspin expression is associated with response to adjuvant 5-fluorouracil based chemotherapy in patients with stage III colon cancer. Int J Cancer 118: 2247-2254, 2006.

20. Wilson CR, Davidson SE, Margison GP, Jackson SP, Hendry JH and West CM: Expression of Ku70 correlates with survival in carcinoma of the cervix. Br J Cancer 83: 1702-1706, 2000.

21. Gu Y, Jin S, Gao Y, Weaver DT and Alt FW: Ku70-deficient embryonic stem cells have increased ionizing radiosensitivity, defective DNA end-binding activity, and inability to support V(D)J recombination. Proc Natl Acad Sci USA 94: 8076-8081, 1997.

22. Li GC, He F, Shao X, Urano M, Shen L, Kim D, Borrelli M, Leibel SA, Gutin PH and Ling CC: Adenovirus-mediated heatactivated antisense $\mathrm{Ku} 70$ expression radiosensitizes tumor cells in vitro and in vivo. Cancer Res 63: 3268-3274, 2003.

23. Celli GB, Denchi EL, de Lange T: Ku70 stimulates fusion of dysfunctional telomeres yet protects chromosome ends from homologous recombination. Nat Cell Biol 8: 885-890, 2006.

24. Gu Y, Seidl KJ, Rathbun GA, Zhu C, Manis JP, van der Stoep N, Davidson L, Cheng HL, Sekiguchi JM, Frank K, StanhopeBaker P, Schlissel MS, Roth DB and Alt FW: Growth retardation and leaky SCID phenotype of Ku70-deficient mice. Immunity 7: 653-665, 1997.

25. Ryu SW, Chae SK and Kim E: Interaction of Daxx, a Fas binding protein, with sentrin and Ubc9. Biochem Biophys Res Commun 279: 6-10, 2000. 